\title{
University of Heidelberg
}

Department of Economics

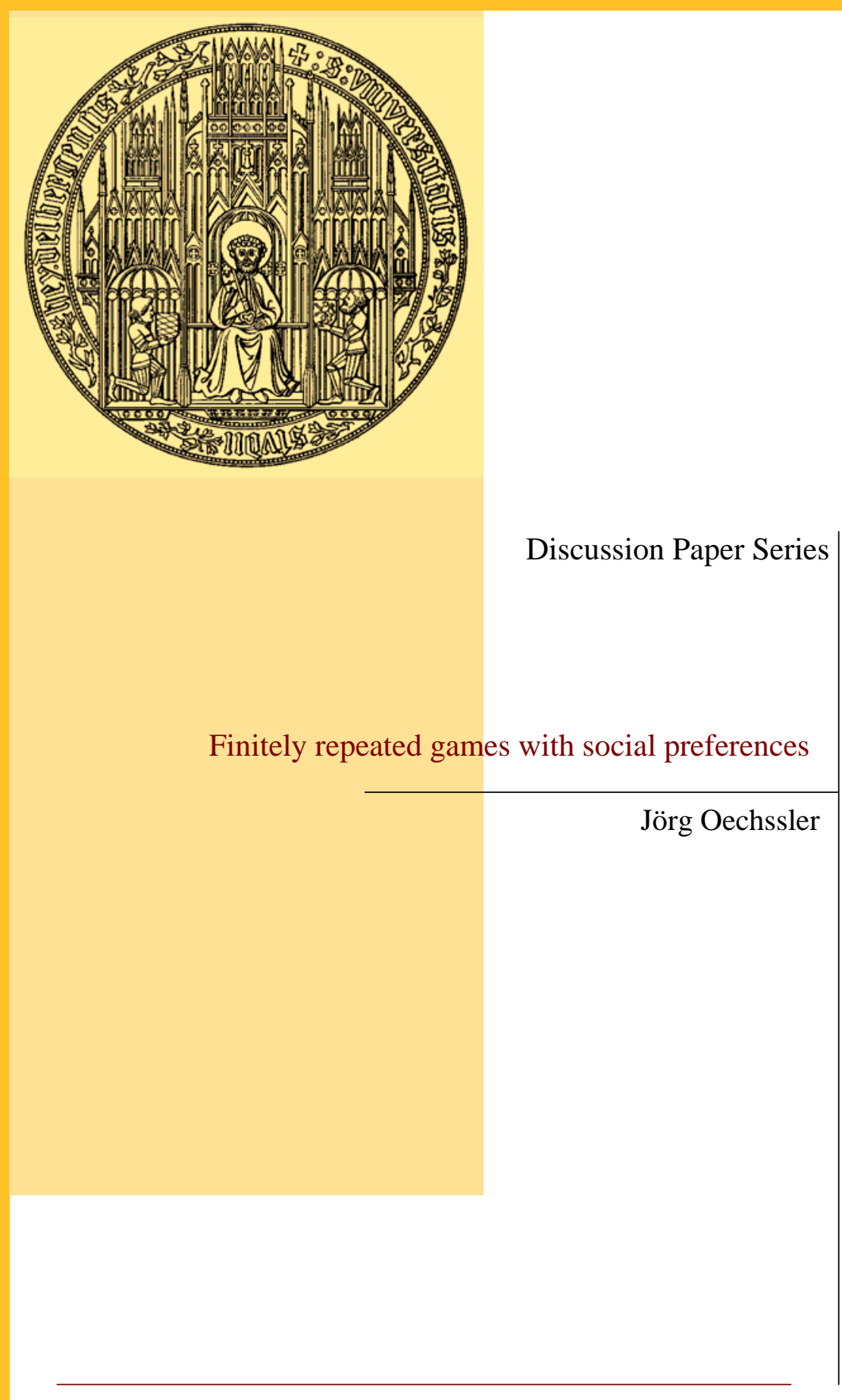

No. 512

April 2011 


\title{
Finitely repeated games with social preferences*
}

\author{
Jörg Oechssler ${ }^{\dagger}$ \\ Department of Economics \\ University of Heidelberg
}

April 4, 2011

\begin{abstract}
A well-known result from the theory of finitely repeated games states that if the stage game has a unique equilibrium, then there is a unique subgame perfect equilibrium in the finitely repeated game in which the equilibrium of the stage game is being played in every period. Here I show that this result does in general not hold anymore if players have social preferences of the form frequently assumed in the recent literature, for example in the inequity aversion models of Fehr and Schmidt (1999) or Bolton and Ockenfels (2000). In fact, repeating the unique stage game equilibrium may not be a subgame perfect equilibrium at all.
\end{abstract}

Keywords: social preferences, finitely repeated games, inequity aversion, ERC.

JEL-Classifications: $\mathrm{C} 72, \mathrm{C} 73$.

\footnotetext{
${ }^{*}$ I thank Dirk Engelmann and Nikos Nikiforakis for discussions and comments.

${ }^{\dagger}$ Email: oechssler@uni-hd.de
} 


\section{Introduction}

A well-known result from the theory of finitely repeated games states that if the stage game has a unique equilibrium, then there is a unique subgame perfect equilibrium in the finitely repeated game in which the equilibrium of the stage game is being played in every period. This result has been much used in applied theory, in particular in industrial organization with the most prominent example being the chain-store paradox of Selten (1980). It is also frequently being invoked in experimental economics when a stage game is played amongst the same set of players (partner or fixed matching) for a finite number of periods.

The purpose of this note is to point out that the result described above does in general not hold anymore if players have social preferences of the form frequently assumed in the recent literature, for example in the inequity aversion models of Fehr and Schmidt (1999) or Bolton and Ockenfels (2000). In fact, repeating the unique stage game equilibrium may not be a subgame perfect equilibrium at all in some examples. The logic is simple. In the standard case of selfish preferences, payoffs are separable across periods in the sense that the optimal choice in the last period does not depend on anything that has happened in previous periods. For most models of social preferences, this no longer holds. What has happened in previous periods influences the relative payoffs and therefore also the optimal choice in the last period, which makes it impossible to treat the last period as independent from the rest of the game. ${ }^{1}$

\section{Examples}

Example 1 (Dictator game) As a simple illustration consider the following example of a dictator game with three options for the proposer: $(0,100)$, $(40,40),(100,0)$, where $x$ in $(x, y)$ denotes the the amount of money allocated to the dictator and $y$ denotes the amount of money allocated to the other player, the recipient. Choose your favorite model of social prefer-

\footnotetext{
${ }^{1}$ In other words, the game is now a stochastic game.
} 
ences and parametrize it such that the proposer ranks $(40,40) \succ(100,0) \succeq$ $(0,100)$. In all examples in this section, preferences depend on average payoffs across periods as is usually assumed in the theory of finitely repeated games. Assume further that preferences are monotone in the sense that $(a, a) \succ(b, b)$ for $a>b$.

Clearly, the unique Nash equilibrium of the stage game is to allocate the money $(40,40)$. However, in the twice repeated game there are two subgame perfect equilibria (SPE) that yield the sequence of allocations $(100,0) \rightarrow$ $(0,100)$ or $(0,100) \rightarrow(100,0)$, respectively. Since $(50,50) \succ(40,40)$, repeating the unique stage game equilibrium twice is not a SPE of the repeated game.

Example 2 (Ultimatum game) This example shows that the inefficiency of the outcome with equal payoffs from the previous example is not a necessary condition. Consider a twice repeated ultimatum game, in which the proposer can make offers of $y \in[0,100]$ to the responder. Suppose the responder is known to be inequity averse as in the Fehr and Schmidt (1999) model such that he will reject any offer $y<30$. For a proposer, who is supposed to care only about his own payoff, the unique best reply is to choose $y=30$. Hence, all SPE of the stage game result in $y=30$, which is accepted by the responder. Yet, in the twice repeated ultimatum game, all offers $y^{1}, y^{2}$ in the two periods with $\left(y^{1}+y^{2}\right) / 2=30$ would be part of a SPE.

Example 3 (Trust game) Consider a 5 times repeated trust game. The stage game payoffs of the investor and the trustee are $\left(\pi_{I}, \pi_{T}\right)=(2,0)$ if the investor does not invest. If he invests, the trustee can split the pie equally $(4,4)$ or keep everything for himself $(0,8)$. Suppose players are inequity averse but not too strongly if it is to their advantage. In particular, suppose that $(2,0) \succ_{I}(0,8),(1.6,1.6) \succ_{I}(2,0),(1.6,1.6) \succ_{I}(a, b)$ if $a<1.6<b$, and $(M-a, a) \succ_{T}\left(M-a^{\prime}, a^{\prime}\right)$ for all $M$ and $a>a^{\prime}{ }^{2}$. The unique subgame perfect equilibrium of the stage game entails no investment. Yet, all subgame perfect equilibria of the 5 times repeated game entail investment in one of

\footnotetext{
${ }^{2}$ This would be the case, for example, in the Fehr and Schmidt (1999) model if $\beta_{i}$ in equation (2) is such that $0.2<\beta_{i}<0.5$ for both players.
} 
the 5 rounds. Suppose there was no investment in the first 4 rounds. By investing in the last round, the investor can equalize average payoffs to $(1.6,1.6)$ which is preferred by him to $(2,0)$, which would result from not investing in the last period.

\section{$3 \quad$ Model and main result}

In the following I shall consider a (normal form) stage game $G=\left\{N,\left(A_{i}\right)\right.$, $\left.\left(\pi_{i}\right)\right\},{ }^{3}$ where $N=1, \ldots, n$ is the set of players, $A_{i}$ is a set of pure actions for player $i$ and $\pi_{i}\left(a_{i}, a_{-i}\right)$ is player $i$ 's payoff function given his action and the action profile of all other players $-i$. An $a \in A:=\times A_{i}$ is referred to as an outcome of $G$. The finitely repeated game $G(T)$ results when $G$ is successively played $T$ times and players are informed about the outcome $a^{t}$ after each period $t$.

The crucial assumption is how players evaluate the outcome sequences $\left(a^{t}\right) \in A^{T}$. The standard assumption in the theory of finitely repeated games is that player $i$ evaluates $\left(a^{t}\right)$ according to his average payoff,

$$
\Pi_{i}:=\frac{1}{T} \sum_{t=1}^{T} \pi_{i}\left(a^{t}\right),
$$

(cf. Benoit and Krishna, 1985, and Osborne and Rubinstein, 1994).

When applying the theory to social preferences, there are (at least) two possibilities of how to evaluate payoffs. Given that in finitely repeated games (and in almost all experiments) the payoffs are paid out to players at the end of the game, it seems reasonable that players should evaluate an outcome sequence $\left(a^{t}\right)$ based on the profile of average payoffs of all players, $\left(\Pi_{i}\right)$.

Assumption 1 Social preferences in a finitely repeated game can be represented by a utility function $U_{i}\left(\Pi_{i}, \Pi_{-i}\right)$.

Likewise, one can assume that $U_{i}$ depends on the sum of payoffs, $\sum_{t=1}^{T} \pi_{i}\left(a^{t}\right)$, which does not affect the main results. The crucial thing is that payoffs

\footnotetext{
${ }^{3}$ With slight modifications the analysis can also be applied to sequential stage games with a unique SPE.
} 
for each player are first aggregated over periods and then compared across players. An alternatively way would be to assume that payoffs are compared period by period without allowing for compensation across periods. This assumption is sometimes made for infinite games (see Duffy and MonozGarcia, 2010), however mainly for reasons of tractability. It seems less reasonable for the case of finitely repeated games. After all, why should a player fail to consider the payoffs from different periods as substitutes when in the end all that matters is the average or total payoff over all periods?

Several prominent social preference models can be applied in accordance with Assumption 1. The inequity aversion model of Fehr and Schmidt (1999) can be specified as

$U_{i}\left(\Pi_{i}, \Pi_{-i}\right)=\Pi_{i}-\alpha_{i} \frac{1}{n-1} \sum_{j \neq i} \max \left[\Pi_{j}-\Pi_{i}, 0\right]-\beta_{i} \frac{1}{n-1} \sum_{j \neq i} \max \left[\Pi_{i}-\Pi_{j}, 0\right]$,

with $0 \leq \beta_{i}<1$ and $\beta_{i} \leq \alpha_{i}$.

The model of Bolton and Ockenfels (2000) can be written as

$$
U_{i}\left(\Pi_{i}, \Pi_{-i}\right)=V_{i}\left(\Pi_{i}, \sigma_{i}\right),
$$

where $\sigma_{i}:=\Pi_{i} / \sum_{j} \Pi_{j}$. Bolton and Ockenfels (2000) assume that $V_{i}$ is strictly concave in $\sigma_{i}$ and assumes a maximum for given $\Pi_{i}$ if $\sigma_{i}=1 / n$. Furthermore, for given $\sigma_{i}, V_{i}$ is strictly increasing in $\Pi_{i}$.

Charness and Rabin (2002) assume a payoff function of the form

$$
U_{i}\left(\Pi_{i}, \Pi_{-i}\right)=(1-\gamma) \Pi_{i}+\gamma\left[\delta \min \left\{\Pi_{1}, \ldots, \Pi_{n}\right\}+(1-\delta) \sum_{j} \Pi_{j}\right] .
$$

Finally, models of linear altruism or spite can be written as

$$
U_{i}\left(\Pi_{i}, \Pi_{-i}\right)=\sum_{j} \gamma_{j} \Pi_{j}
$$

For selfish preferences, the following result is well known (see e.g. Proposition 157.2 in Osborne and Rubinstein, 1994). ${ }^{4}$ It is instructive to follow

\footnotetext{
${ }^{4}$ As Osborne and Rubinstein (1994, Definition 137.1) point out, the crucial assumption for the standard result is "weak separability" of preferences.
} 
the steps of its proof to see why the result breaks down for most forms of social preferences. Suppose Assumption 1 holds. Suppose further that $U(\cdot, \cdot)$ is a linear mapping and hence

$$
\begin{aligned}
U_{i}\left(\Pi_{i}, \Pi_{-i}\right) & =U_{i}\left(\frac{1}{T} \sum_{t=1}^{T} \pi_{i}\left(a^{t}\right), \frac{1}{T} \sum_{t=1}^{T} \pi_{-i}\left(a^{t}\right)\right) \\
& =\frac{1}{T} \sum_{t=1}^{T} U_{i}\left(\pi_{i}\left(a^{t}\right), \pi_{-i}\left(a^{t}\right)\right) .
\end{aligned}
$$

Obviously, (6) is satisfied if $U_{i}$ is given by (1) or (5) but not if it is given by (2), (3), or (4). This is the reason why for most of the popular social preference models the following proposition does not hold.

Proposition 1 Suppose payoffs in $G(T)$ are evaluated by a utility function $U_{i}\left(\Pi_{i}, \Pi_{-i}\right)$ that satisfies (6). If the strategic game $G$ has a unique Nash equilibrium payoff profile, then for any value of $T$ the action profile chosen after any history in any subgame perfect equilibrium of $G(T)$ is a Nash equilibrium of $G$.

Proof. Consider the optimal action in period $T$. If (6) holds, then

$$
\begin{aligned}
\arg \max _{a_{i}^{T}} U_{i}\left(\Pi_{i}, \Pi_{-i}\right) & =\arg \max _{a_{i}^{T}} \frac{1}{T} \sum_{t=1}^{T} U_{i}\left(\pi_{i}\left(a^{t}\right), \pi_{-i}\left(a^{t}\right)\right) \\
& =\arg \max _{a_{i}^{T}} U_{i}\left(\pi_{i}\left(a^{T}\right), \pi_{-i}\left(a^{T}\right)\right) .
\end{aligned}
$$

The optimization problem in the second line is the same as the one in the stage game. This implies that players' payoffs in $T$ are independent of the history of play. Thus, in all subgames of $G(T)$ starting in period $T$, the outcome is a Nash equilibrium of $G$. Therefore, also in all subgames starting in period $T-1$, the outcome is a Nash equilibrium of $G$. The result follows then by induction.

If $U(\cdot, \cdot)$ is non-linear (e.g. because of the strict concavity with respect to $\sigma_{i}$ in the model of Bolton and Ockenfels, 2000, or the max-operators in the model of Fehr and Schmidt, 1999), the naive application of the backwardinduction argument fails. 
Thus, when applying social preference models to finitely repeated games, care has to be taken. It will depend on the specifics of the game whether the $T$-fold repetition of the unique stage game equilibrium is still a SPE of the repeated game. Uniqueness of SPE will in general be lost.

\section{References}

[1] Benoit, Jean-Pierre and Krishna, Vijay (1985), "Finitely Repeated Games", Econometrica, 53, 905-922

[2] Bolton, Gary E. and Ockenfels, Axel (2000), "A theory of equity, reciprocity and competition", American Economic Review 100, 166-193.

[3] Charness, Gary and Rabin, Matthew (2002), "Understanding Social Preferences with Simple Tests", Quarterly Journal of Economics, 117, 817-869.

[4] Duffy, John and Muñoz-García, Félix (2010), "Patience or Fairness? Analyzing Social Preferences in Repeated Games", mimeo University of Pittsburgh.

[5] Fehr, Ernst and Schmidt, Klaus M. (1999), "A Theory of Fairness, Competition and Cooperation." Quarterly Journal of Economics 114, 817868.

[6] Osborne, Martin and Ariel Rubinstein (1994). A course in game theory, MIT Press. 